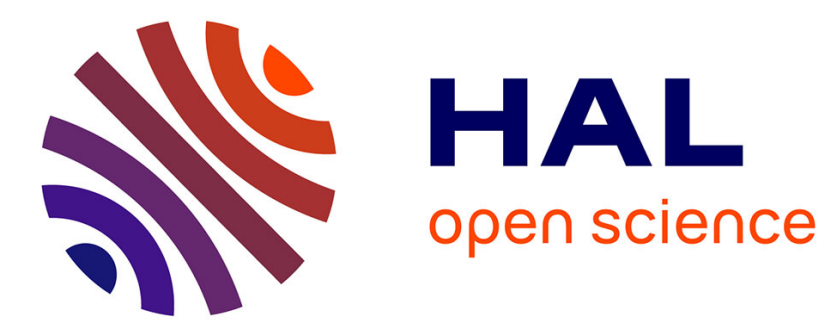

\title{
Self-organized UAV-based Supervision and Connectivity: Challenges and Opportunities
}

Yann Busnel, Christelle Caillouet, David Coudert

\section{To cite this version:}

Yann Busnel, Christelle Caillouet, David Coudert. Self-organized UAV-based Supervision and Connectivity: Challenges and Opportunities. NCA 2019 - 18th IEEE International Symposium on Network Computing and Applications, Sep 2019, Cambridge, United States. pp.1-5, 10.1109/NCA.2019.8935060 . hal-02267396

\section{HAL Id: hal-02267396 https://hal.inria.fr/hal-02267396}

Submitted on 19 Aug 2019

HAL is a multi-disciplinary open access archive for the deposit and dissemination of scientific research documents, whether they are published or not. The documents may come from teaching and research institutions in France or abroad, or from public or private research centers.
L'archive ouverte pluridisciplinaire HAL, est destinée au dépôt et à la diffusion de documents scientifiques de niveau recherche, publiés ou non, émanant des établissements d'enseignement et de recherche français ou étrangers, des laboratoires publics ou privés. 


\title{
Self-organized UAV-based Supervision and Connectivity: Challenges and Opportunities
}

\author{
Yann Busnel $^{*}$, Christelle Caillouet ${ }^{\dagger}$, David Coudert ${ }^{\dagger}$ \\ ${ }^{*}$ IMT Atlantique, IRISA, CNRS UMR 6074 \\ †Université Côte d'Azur, Inria, I3S, CNRS, France
}

\begin{abstract}
The use of drones has become more widespread in recent years. Many use cases have developed involving these autonomous vehicles, ranging from simple delivery of packages to complex emergency situations following catastrophic events. The miniaturization and very low cost of these machines make it possible today to create large meshes to ensure network coverage in disaster areas, for instance. However, the problems of scaling up and self-organization are necessary to solve problems in these use cases. This position paper first presents different new requirements for the deployment of unmanned aerial vehicles (UAV) networks, involving the use of many drones. Then, it introduces solutions from distributed algorithms and real-time data processing to ensure quasi-optimal solutions to the raised problems.
\end{abstract}

\section{INTRODUCTION}

Autonomous air systems extend the reach of conventional wireless networks by providing supervision and coverage solutions for wide area monitoring, healthcare management, or post-disaster management [1], [25], [36]. The density and node mobility are no longer constrained because the air system makes it possible to provide network connectivity and services and expand on-demand deployments for data recovery, for tracking mobile users or to recharge the batteries of the nodes using technologies such as radio frequency [16]. The use of a single drone in an autonomous air system does not allow to generically respond to all the temporal and spatial constraints of sensor networks. On the other hand, the use of a fleet of drones would help to overcome these constraints. However, the use of a fleet in an autonomous air system presents several challenges in terms of coordination between drones, air-toground data transfer and air-to-air, and dynamic coverage for energy recharging or connectivity servicing for instance [38].

The drones must be optimally positioned above the ground nodes to be able to cover a large area while minimizing the deployment cost of the drones and the energy cost spent by each drone. The three-dimensional space in which the drones are evolving complicates the optimization models, as they have to take into account the quality constraints of air-to-ground and air-to-air communications (see Figure 1).

The autonomous air system must be able to retrieve data from ground-based nodes and transfer them to a base station located on the edge or the center of the network. Network connectivity for air-to-ground and air-to-air communications must be ensured by the deployment while maintaining the cost constraints spent by the autonomous air system, and guaranty efficient data gathering and transfer to cloud services.

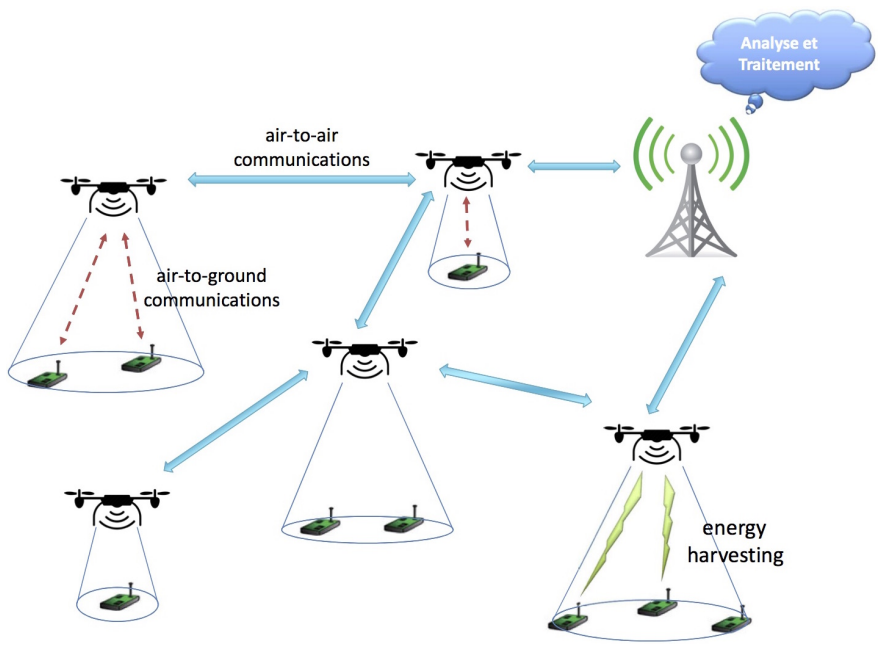

Fig. 1: Multi-hop data collection using UAVs

However, in the context of networks deployed on a very large scale, or even in multi-site, the collection and processing of this data is delicate and ambitious. Over the past two decades, and more recently with the advent of Big Data issues, the need to process large amounts of information in potentially distributed, large-scale data streams has become paramount. Relying on traditional information processing algorithms is not feasible in a real-time context, with very low storage and processing capacity. Streaming algorithms have shown their highly desirable properties in data intensive monitoring applications [8]. These algorithms process the input stream in a single pass and sequentially. All these algorithms rely on pseudo-random functions that map elements of the stream to uniformly distributed image values. The interested reader is invited to read the nice survey by Muthukrishnan [41].

To the best of our knowledge, the above techniques have not yet been included in the supervision tools via unmanned aerial vehicles (UAVs). It therefore seems to us relevant, even necessary, to determine which tools and techniques from multiple domains could be candidates for automatic analysis in order to improve the relevance of the data to be transmitted, on the one hand, and on the other hand, which aggregates or samples could be considered to optimize the complexity of communication.

The necessary multi-topic openness offers a great opportunity to cooperate with specialists from different fields in 
order to bring them to work on the relationship between their work, in some innovative global projects. To our knowledge, collaboration on the frontier of these themes seems relevant and not yet addressed. We present below various problems that need to be addressed in this area.

\section{Challenging PROBlems}

Data collection is an important mechanism for making wireless sensor networks viable. Using UAVs as communication relays have got much attention in recent years to bridge communication between nodes on the ground and to provide the best communication facilities to the participating base stations. The problem of collecting aerial data (ADCP) from a set of fixed or mobile wireless nodes located on the ground, using a fleet of drones, has received a lot of interest recently [11], [12], [13], [14]. The objective is to deploy a set of unmanned aerial vehicles (UAVs) in 3D space to cover and collect data from all mobile wireless nodes at each stage of ground-toair communication, to send this data to a central base station connected to cloud services using multi-hop wireless air-to-air communications via the UAV network, to reduce the total cost of deployment (communication and deployment) over time. ADCP is a complex problem of temporal and spatial coverage and connectivity. The authors proposed several solutions, allowing them to be adapted to the size of the network to be supervised. They obtained either optimal solutions using linear programming with decomposition methods and column generation approaches, or approximated schemes using distributed algorithms. The results show that their approach provides very specific solutions that minimize data collection costs. On the one hand the optimal UAV planning gives insights about the number of necessary drones for ADCP, and the altitude at which they should be placed to optimize the data gathering. On the other hand, the distributed solutions allows the drones to autonomously discover the ground users and continuously report event information observed to the base station. The connectivity of the deployed UAVs with the base station is continuously maintained throughout the deployment process.

This work proposes to address the barrier of scaling up ADCP and optimize data communications. We show that this is a main issue in several application domains and remains under addressed in the literature to the best of our knowledge.

\section{A. Monitoring applications}

Agriculture and animal health monitoring and tracking are recently attracting more attention. Sensors are placed in the field to capture information related to the quality of the crops and the well-being of animals [46], or to track wild animals in Africa [43] or sharks near the Reunion Island in the Indian Ocean. In such environments, the sensors do not wake up very often and store the regularly measured data in their memory. A fleet of drones is particularly of interest to provide an operational network to collect periodically data of high volume from the sensors and gather it to the base station. Zorbas and O'Flynn considered in [46] that animal monitoring can generate a high volume of data (of several MBytes within a week) due to the accelerator data that are continuously generated when the animals are moving. To tackle the issue of the high data volume transfer, they propose a network architecture combining different communication technologies, like LoRa and WiFi.

Another approach could consist in analyzing precisely the data streams and cut them up into samples. Each sample is associated with a specific priority so that the use of UAV is optimized. In such a way, the drones can periodically fly over the ground nodes and collect in priority the most important data. Optimizing both the use of the drones and the sampling of the volume of data is the key objective of our approach. This could improve the solution of [46] by reducing the amount of data to be forwarded by the UAVs, thus enhancing their efficiency and decreasing their flying time.

\section{B. Security}

An important field of application for drones concerns natural disasters such as earthquakes, tsunamis, tropical storms, fires, etc. [36]. The effects of these large-scale natural disasters could be significantly mitigated through a comprehensive, and highly efficient disaster management system. In such situations, time is a critical factor in finding survivors, and it is important to provide connectivity through a quick and effective solution to rescue teams. Unless traditional ground emergency systems that are usually low efficient in information gathering with large latency and low bandwidth satellite communications, the use of UAVs has become a key solution to enhance the capability of disaster responses, deal with the extreme environmental conditions without any network connectivity and conduct timely rescue work. For instance, drones have been designed to leverage smart devices owned by the survivors in devastated areas and provide an on-demand communication infrastructure for rescue operations [38]. Autoorganization through an effective path planning of the drone fleet in a decentralized way is the top priority study for providing an efficient disaster management system.

Military battlefield management is of particular interest, and their requirements are similar to the one presented above for civil disaster management scenarios. Moreover, the accuracy and security of information is also an important issue. The flying system must guarantee the transmission of information that can be prioritized, but also redundant. Thus, limiting multiple transmissions of the same information would be of great help in accelerating rescue operations. A complementary requirement is to prevent information to be received by external nodes in order to guarantee data security and prevent attacks. The analysis of the data stream to limit this redundancy and prevent malicious attacks is of great interest in using drones for such applications.

\section{Energy consuption and harvesting}

Networking technologies are essential to the success of UAV deployment in practical environments. Due to their low computing and storage facilities, it is difficult for them to integrate all these used technologies : Bluetooth, 802.11 Wi-Fi, 
2G/3G/4G LTE cellular network, low power (LoRa, Sigfox), GPS assisted satellite. The choice of embedded technologies strongly depends on the application. For instance, military battlefield management usually requires satellite communications with control services. On the contrary, rescuing operations have to adapt to the equipment owned by the survivors, usually cellular smartphones with additional features like Wifi and Bluetooth. Selecting which technology to install on the drones and use for energy efficient communications with the ground has been investigated in [39].

The volume of data to be exchanged can vary a lot (small messages, video streaming, ...) and impact energy consumption of the nodes and drones. Limiting the amount of transferred data using caching and stream analysis is a key challenge to ensure connectivity to the ground nodes in white areas or post-disaster conditions. The energy perspective has to be investigated to balance the effect of data exchange, flying trajectory of the drones, and rescue time.

The drones can also, thanks to a radio-frequency system, recharge the batteries of ground-based nodes. This technology has recently been studied for autonomous aerial systems [15], [16]. Results show the effectiveness of the approach for extending the life of the sensors on the ground at the same time as monitoring and collection. The recharge adds the temporal dimension as a strong constraint in the deployment. The sensors require the presence of drones at a given location (proximity) for a given time (recharging time). UAVs must also take into account the battery level of each node to optimize recharging while minimizing the number and the associated energy costs of deployed drones. Robustness of the recharge has also to be assessed by considering the survival of all or part of the ground-based network, as well as in the reliability of radio frequency communications dedicated to recharging. In this context, the transmitted power, recharging efficiency, packet throughput, interference, the position of the drones/sensors and many other parameters have to be taken into account.

In each of these scenarios, it is important to coordinate the exchange of messages in order to ensure that the nodes on the ground are recharged, limit the energy consumption of communicating objects, and ensure that information is efficiently transmitted between services and users.

\section{Health related information}

New applications related to medicine and healthcare have appeared recently. Drones have been set to deliver medicine, medical supplies such as defibrillators, blood samples, and vaccines in east Africa [1]. These new healthcare delivery systems involve logistic requirements as well as guarantees in the accuracy of the information. Malicious attacks have to be prevented by detecting patterns that do not conform to the expected behaviour and make the drone changes its original path plan. Determining and maintaining a safe route based on the terrain, weather, airspace and population density is a key challenge. The route should also be computed efficiently to avoid white areas such as airports and schools. A new constraint appears in such systems according to the delivery requirement, corresponding to the weight of the carried supplies by the UAVs. The miniaturization and very low cost of these machines enforce to limit the amount and weight of the carried objects. In this context, the trajectory of the UAV has to be optimized to guaranty a total flying time, a specific altitude, and a maximum total weight.

Optimization techniques and decentralized algorithms have to be developed to combine all these new requirements. To the best of our knowledge, these applications have not been much addressed in the literature so far.

\section{Methodology}

To compensate for the lack of computing power of UAVs, it is customary for all calculations to be centralized, in a remote cloud for example. However, this solution is not viable, on the one hand in terms of energy consumption linked to air-to-air communication and also because of the wireless bandwidth which will eventually become a bottleneck for data-intensive communication. One of the key points of the new solutions will be to use the unused memory on the drones, as well as local computing power, which often generates less power consumption. The objective is to build a decentralized system, or at least a hybrid ad-hoc one, that scales elegantly.

\section{A. Distributed Algorithms for self-organization}

To be useful, this ad-hoc distributed computing environment must surpass, either in latency or battery consumption, two alternative approaches: strictly local computation and the delegation of data to the cloud services on the Internet.

The ever growing success of systems and applications built on the peer-to-peer approach lead in the recent past to a vast effort for creating algorithms that could support the development of such applications. One of the major problems that must be solved in the design phase of any decentralized system is how to build and maintain an application-level network that links together participating processes and that can be used to communicate and inter-operate. Such a network is often referred to as an overlay network, because it represents a generic application level network that is superimposed over an existing network (either logical or physical). Many algorithms have been proposed to build and maintain such structures in very different settings; each solution produces networks with different topologies and different performance characteristics [35], [32]. Moreover, most large scale systems have been usually designed to run a single specific application. Shifting the view from ad-hoc designs to general overlay management protocols that can be used to build and maintain large scale overlay networks on top of which several different distributed protocols can concurrently run raises the need for methods able to track the distribution of available resources. As an example it could be useful to divide clients participating to a large overlay in groups depending on their computational power, such that different running algorithms can fairly share the most powerful nodes. Existing approaches are mainly based on centralized information gathering and manual allocation of 
resources; such approaches, however, hardly scale with the size of the system [27].

One of the relevant approaches in the development of distributed algorithms is the epidemic protocol model. Initially introduced to disseminate information, epidemic protocols are based on a very simple but very reliable and effective paradigm. Originally, this paradigm was proposed as part of database maintenance [20]. Since then, many studies have extended this model and the term epidemic protocol (aka gossip-based protocol) is now also used for any probabilistic protocol, based on periodic exchanges of information between participants [21]. Since then, they have been applied in many settings in wired and wireless systems as in [10], [22], [24], [26], [44], [45].

The basic principle of this type of protocol is refined: each participant has a set of information that he/she makes available to others, and has the possibility to publish new ones. The objective of each participant is to collaborate in order to obtain up-to-date information on the network (for example, in [20], to know all the updates of the database). Each node periodically selects another node in the network, with which it exchanges information. According to this communication paradigm, all the nodes in the system have all the information available in a very short time. Thus, the propagation of information behaves in the same way as the propagation of epidemia in a network of human interaction [42] (e.g., virus, rumour, etc.). A generic framework has been proposed in [28] to provide a generic substrate for gossip peer sampling protocols, providing a common ground for membership systems. In this paper, the authors explore the resulting topologies and show that the parameters of the generic gossip protocols can be set to achieve random-like graph topologies i.e. providing each node with a random sample of the network.

\section{B. The value of the stream model}

In some use case ( $c f$. , Sections II-B or II-D), it is necessary to process a considerable amount of data quickly and accurately. However, the low memory capacities made available for these monitoring and metrology operations make the problem very ambitious and particularly complex. The diversity and dynamic nature of content therefore requires the proposal of innovative real-time solutions with low complexity, in terms of memory, processing time, communication, etc. [41].

The problem of extracting relevant information from a (distributed) data stream(s) is similar to the problem of identifying patterns that do not conform to the expected behaviour. This theme has been a very productive field of research in recent decades. For example, depending on the specificities of the domain and the type of anomalies considered, different methods have been designed: classification, grouping, nearest neighbour, spectral statistics and information theory. An interesting state of the art on these techniques, describing their advantages and disadvantages, is proposed by [18]. A common feature of these techniques is their high complexity in terms of space and computation cost, because they are based on algorithms "with unbounded memory", i.e., requiring complete knowledge and access to the analyzed data.

Relying on algorithms of this type is not feasible in a context of real-time information extraction, with very low storage and processing capacity. This is particularly the case for the application considered in Section II. Also, there are two main approaches for real-time processing of massive, potentially distributed data flows. The first consists in regularly sampling the flow, making it possible to limit the amount of data stored in memory [2], [30], [31], [33]. This method allows to calculate exactly functions on these samples. However, the reliability of this calculation, in comparison with the exact result calculated on the entire flow, depends strongly on the volume of data that has been sampled, and the position of this data on the stream. Worse still, an opponent could easily take advantage of the sampling policy to hide his attacks among the unsampled packets, or thereby prevent his "malicious" packets from being correlated. However, recent work has made it possible to guarantee resilience to attacks while ensuring the detection of frequent events [5].

On the other hand, the second approach consists in processing all the data in the stream on the fly, and keeping locally only summaries, or aggregates, in which only essential information about the data is kept [18]. This approach extracts statistics on processed data streams, with bounded error probabilities, without assuming any constraint on the order in which the data is received on the nodes (i.e., the order of the data could be manipulated by an omnipotent adversary [6]). Most existing research using this approach has focused on calculating statistical functions or measurements with a given $\varepsilon$ error, using a poly-logarithmic memory amount in the size of the stream and the domain of the incoming data. These functions estimate, for example, the number of distinct data present in a given stream [9], [23], [29], the frequency moments [2], the most frequent elements [2], [19], [40], [37], the entropy of a flow [17], [34], the co-variance between several streams [3] or the relative entropy between a biased stream and a uniform stream [7], [4].

\section{CONCLUSION}

In this paper, we have shown that, thanks to recent improvements in drone design and cost reduction, fleets of UAVs are nowadays becoming credible candidates for many applications from landscape monitoring to complex emergency situations following catastrophic events. Furthermore, we have identified research challenges for solving the problems of scaling up and self-organization that could slow down the adoption of UAVs in practice. However, we have shown that the fields of distributed algorithms and real-time data processing have proposed a variety of solutions that can be used to ensure near-optimal operation of fleets of UAVs, and hence leverage and foster the use of UAVs in these use cases. This gets rise to promising research and development opportunities.

\section{REFERENCES}

[1] E. Ackerman and E. Strickland. Medical delivery drones take flight in east africa. IEEE Spectrum, 55(1):34-35, January 2018. 
[2] N. Alon, Y. Matias, and M. Szegedy. The space complexity of approximating the frequency moments. In Proceedings of the 28th ACM Symposium on Theory of computing (STOC), pages 20-29, 1996.

[3] E. Anceaume and Y. Busnel. Deviation estimation between distributed data streams. In Proceedings of the 10th European Dependable Computing Conference, Newcastle (EDCC), pages 35-45, 2014.

[4] E. Anceaume and Y. Busnel. A distributed information divergence estimation over data streams. IEEE Transaction on Parallel and Distributed System, 25(2):478-487, 2014.

[5] E. Anceaume, Y. Busnel, and V. Cazacu. On the Fly Detection of the Top-k Items in the Distributed Sliding Window Model. In $N C A$ 2018 - 17th IEEE International Symposium on Network Computing and Applications, pages 1-8, Boston, United States, Nov. 2018. IEEE, IEEE.

[6] E. Anceaume, Y. Busnel, and S. Gambs. Uniform and Ergodic Sampling in Unstructured Peer-to-Peer Systems with Malicious Nodes. In Proceedings of the 14th International Conference on Principles of Distributed Systems (OPODIS), volume 6490, pages 64-78, 2010.

[7] E. Anceaume, Y. Busnel, and S. Gambs. Ankle: Detecting attacks in large scale systems via information divergence. In Proceedings of the 9th European Dependable Computing Conference (EDCC), 2012.

[8] E. Anceaume, Y. Busnel, N. Rivetti, and B. Sericola. Identifying global icebergs in distributed streams. In Proceedings of the 34th IEEE Symposium on Reliable Distributed Systems (SRDS), pages 266-275, 2015.

[9] Z. Bar-Yossef, T. S. Jayram, R. Kumar, D. Sivakumar, and L. Trevisan. Counting distinct elements in a data stream. In Proceedings of the 6th International Workshop on Randomization and Approximation Techniques (RANDOM), pages 1-10. Springer-Verlag, 2002.

[10] Y. Busnel, M. Bertier, E. Fleury, and A.-M. Kermarrec. GCP: Gossipbased code propagation for large-scale mobile wireless sensor networks. In 1st International Conference on Autonomic Computing and Communication Systems (Autonomics '07), page 11, Rome, Italie, octobre 2007.

[11] Y. Busnel, C. Caillouet, and D. Coudert. VESPA: Constrained target coverage by distributed deployment of connected UAVs. Research report, Inria ; I3S, Université Côte d'Azur ; IMT Atlantique, May 2019.

[12] C. Caillouet, F. Giroire, and T. Razafindralambo. Optimization of mobile sensor coverage with UAVs. In 11th International Workshop on Wireless Sensor, Robot and UAV Networks (WiSARN) in conjunction with IEEE INFOCOM 2018, Honolulu, Hawai, United States, Apr. 2018. IEEE.

[13] C. Caillouet, F. Giroire, and T. Razafindralambo. Efficient Data Collection and Tracking with Flying Drones. Ad Hoc Networks, 89(C):35-46, 2019.

[14] C. Caillouet and T. Razafindralambo. Efficient deployment of connected unmanned aerial vehicles for optimal target coverage. In 8th Global Information Infrastructure and Networking Symposium (GIIS). IEEE, Oct. 2017.

[15] C. Caillouet, T. Razafindralambo, and D. Zorbas. Recharging wireless sensor networks using drones and wireless power transfer. In IEEE PIMRC 2018, Recent Results track.

[16] C. Caillouet, T. Razafindralambo, and D. Zorbas. Optimal placement of drones for fast sensor energy replenishment using wireless power transfer. In Wireless Days 2019, Apr. 2019.

[17] A. Chakrabarti, G. Cormode, and A. McGregor. A near-optimal algorithm for computing the entropy of a stream. In Proceedings of the 18th ACM-SIAM Symposium on Discrete Algorithms (SODA), pages 328-335, 2007.

[18] V. Chandola, A. Banerjee, and V. Kumar. Anomaly detection: A survey. ACM Computing Surveys, 41(3):1-58, 2009.

[19] M. Charikar, K. Chen, and M. Farach-Colton. Finding frequent items in data streams. Theoretical Computer Science, 312(1):3-15, 2004.

[20] A. Demers, D. Greene, C. Hauser, W. Irish, J. Larson, S. Shenker H. Sturgis, D. Swinehart, and D. Terry. Epidemic Algorithms for Replicated Database Maintenance. In Proc. of the 6th Annual Symposium on Principles of Distributed Computing (PODC), pages 1-12. ACM Press, 1987.

[21] P. T. Eugster, R. Guerraoui, A.-M. Kermarrec, and L. Massoulié Epidemic information dissemination in distributed systems. IEEE Computer, 37(5):60-67, mai 2004.

[22] P. T. Eugster, S. Handurukande, R. Guerraoui, A.-M. Kermarrec, and P. Kouznetsov. Lightweight probabilistic broadcast. ACM Transactions on Computer Systems, 21(4):341-374, novembre 2003.

[23] P. Flajolet and G. N. Martin. Probabilistic counting algorithms for data base applications. Journal of Computer and System Sciences, 31(2):182209, 1985
[24] D. Gavidia, S. Voulgaris, and M. van Steen. Epidemic-style monitoring in large-scale wireless sensor networks. Rapport de Recherche IR-CS012.05, Vrije Universiteit Amsterdam, Amsterdam, Hollande, 2005.

[25] L. Gupta, R. Jain, and G. Vaszkun. Survey of important issues in uav communication networks. IEEE Communications Surveys Tutorials, 18(2):1123-1152, Secondquarter 2016.

[26] M. Jelasity and O. Babaoglu. T-man: Gossip-based overlay topology management. In Engineering Self-Organising Systems, volume 3910 of Lecture Notes in Computer Science, pages 1-15. Springer, 2006.

[27] M. Jelasity and A.-M. Kermarrec. Ordered slicing of very large-scale overlay networks. In A. Montresor, A. Wierzbicki, and N. Shahmehri, editors, Peer-to-Peer Computing, pages 117-124. IEEE Computer Society, 2006.

[28] M. Jelasity, S. Voulgaris, R. Guerraoui, A.-M. Kermarrec, and M. van Steen. Gossip-based peer sampling. ACM Trans. Comput. Syst., 25(3):8, août 2007.

[29] D. M. Kane, J. Nelson, and D. P. Woodruff. An optimal algorithm for the distinct element problem. In Proceedings of the 29th ACM Symposium on Principles of Database Systems (PODS), 2010.

[30] V. Karamcheti, D. Geiger, Z. Kedem, and S. Muthuskrishnan. Detecting malicious network traffic using inverse distribution of packet contents. In Proceedings of the 1st Workshop on Mining Network Data (MineNet), 2005.

[31] B. Krishnamurthy, S. Sen, Y. Zhang, and Y. Chen. Sketch-based change detection: Methods, evaluation, and applications. In Proceedings of the 3rd ACM SIGCOMM Internet Measurement Conference (IMC), pages 234-247, 2003.

[32] J. Kurian and K. Sarac. A survey on the design, applications, and enhancements of application-layer overlay networks. ACM Comput. Surv., 43:5:1-5:34, December 2010.

[33] A. Lakhina, M. Crovella, and C.Diot. Mining anomalies using traffic feature distributions. In Proceedings of the 28th ACM Conference on Applications, Technologies, Architectures, and Protocols for Computer Communications (SIGCOMM), 2005.

[34] A. Lall, V. Sekar, M. Ogihara, J. Xu, and H. Zhang. Data streaming algorithms for estimating entropy of network traffic. In Proceedings of the 27th joint International conference on Measurement and modeling of computer systems (SIGMETRICS). ACM, 2006.

[35] E. K. Lua, J. Crowcroft, M. Pias, R. Sharma, and S. Lim. A Survey and Comparison of Peer-to-Peer Overlay Network Schemes. IEEE Communications Surveys and Tutorials, 7:72-93, 2005.

[36] C. Luo, W. Miao, H. Ullah, S. McClean, G. Parr, and G. Min. Unmanned Aerial Vehicles for Disaster Management, pages 83-107. 082019.

[37] A. Metwally, D. Agrawal, and A. El Abbadi. Efficient computation of frequent and top-k elements in data streams. In Proceedings of the 10th International Conference on Database Theory (ICDT), 2005.

[38] F. Mezghani and N. Mitton. Open problem: Energy-and time-efficient dynamic drone path planning for post-disaster network servicing. In ODS 2018 - International Conference on Optimization and Decision Science, Taormina, Italy, Sept. 2018.

[39] F. Mezghani and N. Mitton. Opportunistic Disaster Recovery. Internet Technology Letters, pages 1-5, Jan. 2018.

[40] J. Misra and D. Gries. Finding repeated elements. Science of Computer Programming, 2(2):143-152, 1982.

[41] S. Muthukrishnan. Data Streams: Algorithms and Applications. Now Publishers Inc., 2005.

[42] E. Rivière and P. Gauron. Rechercher parmi ses pairs ou quand le hasard ne fait pas si bien les choses, tutoriel. In 3ème MAnifestation des JEunes Chercheur francophones dans les domaines des STIC (MajecSTIC '05), Rennes, France, novembre 2005

[43] V. Toldov, J. Meijers, R. Igual-Pérez, R. Wolhuter, N. Mitton, and L. Clavier. Performance evaluation of LoRa radio solution for PREDNET wildlife animal tracking project. In LPWAN 2016, Paris Roissy, France, May 2016.

[44] R. van Renesse. Power-aware epidemics. In 21th IEEE Symposium on Reliable Distributed Systems (SRDS '02), pages 358-361, Suita, Japon, octobre 2002

[45] S. Voulgaris, D. Gavidia, and M. van Steen. Cyclon: Inexpensive membership management for unstructured $\mathrm{p} 2 \mathrm{p}$ overlays. Journal of Network System Management, 13(2):197-217, juin 2005.

[46] D. Zorbas and B. O'Flynn. A network architecture for high volume data collection in agricultural applications. In 1st International Workshop on Wireless sensors and Drones in Internet of Things (Wi-DroIT), in conjunction with DCOSS, Santorini Island, Greece, May 2019. 